\title{
A comparison of exact TM plane wave diffraction by coated wedges and impedance wedges
}

Andersen, Lars S.; Breinbjerg, Olav; Moore, John T.

Published in:

Antennas and Propagation Society International Symposium AP-S. Digest

Link to article, DOI:

10.1109/APS.1996.549645

Publication date:

1996

Document Version

Publisher's PDF, also known as Version of record

Link back to DTU Orbit

Citation (APA):

Andersen, L. S., Breinbjerg, O., \& Moore, J. T. (1996). A comparison of exact TM plane wave diffraction by coated wedges and impedance wedges. In Antennas and Propagation Society International Symposium AP-S. Digest (Vol. Volume 1, pp. 494-496). IEEE. https://doi.org/10.1109/APS.1996.549645

\section{General rights}

Copyright and moral rights for the publications made accessible in the public portal are retained by the authors and/or other copyright owners and it is a condition of accessing publications that users recognise and abide by the legal requirements associated with these rights.

- Users may download and print one copy of any publication from the public portal for the purpose of private study or research.

- You may not further distribute the material or use it for any profit-making activity or commercial gain

- You may freely distribute the URL identifying the publication in the public portal 


\title{
A comparison of exact TM plane wave diffraction by coated wedges and impedance wedges
}

\author{
Lars S. Andersen" and Olav Breinbjerg \\ Electromagnetics Institute, Technical University of Denmark, \\ DK-2800 Lyngby, Denmark \\ John T. Moore \\ DEMACO, Champaign, Illinois, USA
}

\section{INTRODUCTION}

In scattering analysis, a perfectly electrically conducting (PEC) structure coated by a dielectric layer can be modelled by a structure at the surface of which the standard impedance boundary condition (SIBC) $[1 ;(5)]$ is enforced. The SIBC is exact for a normally illuminated infinite coated plane but approximate for oblique illumination or curved structures. A coated edge has an infinite curvature and the SIBC approximation is thus not formally applicable. However, coated structures with edges are of practical importance. Some accurate numerical results for SIBC models of such structures have been reported [1]. Furthermore, high-frequency techniques for the analysis of SIBC edge diffraction already exist $[2,3]$. The purpose of this work is to numerically investigate the accuracy of the SIBC approximation for edge diffraction. To this end, we compare the scattering by coated wedges and SIBC wedges for which the diffracted field from a single edge can be observed without interference from direct fields or reflected fields. Results have been obtained in the case of illumination by a transverse magnetic (TM) uniform plane wave. The analysis of the coated wedge is based on an integral equation formulation combined with a hybrid technique, while the analysis of the SIBC wedge is based on Maliuzhinets' solution [4]. Comparisons have been carried out for a series of configurations including lossy coatings as well as lossless coatings permitting unattenuated propagation of surface waves (SW's). The results presented herein show that the presence of an edge in a coated structure does not necessarily make the SIBC approximation inapplicable.

To the knowledge of the authors, a comparison of the edge diffraction by coated wedges and the corresponding SIBC wedges has not previously been reported. Vasil'ev and Solodukhov [5] compared physical optics (PO) results and integral equation results for transverse electric (TE) plane wave scattering by a coated wedge. The integral equations were formulated for a multilayer lossy coating and unattenuated SW propagation was excluded by considering only lossy coatings. Jin and Liepa [6] combined the finite element method (FEM) and PO to numerically determine the exact impedance of a coated wedge for TM plane wave illumination. Unattenuated SW propagation was not considered. Gérard et al. employed an integral equation formulation to determine the diffraction by coated wedges for TE and TM plane wave illumination. The configuration for which numerical results are presented does not include SW's.

\section{FORMULATION OF INTEGRAL EQUATIONS FOR A COATED WEDGE}

We consider a PEC wedge coated by a dielectric layer of thickness d (Fig. 1). The ambient free space region, denoted $R_{0}$, has the permittivity $\varepsilon_{0}$ and the permeability $\mu_{0}$. The coating, denoted $R_{1}$, is electromagnetically characterized by the (possibly complex) permittivity $\varepsilon_{r} \varepsilon_{0}$ and the (possibly complex) permeability $\mu_{\mathrm{r}} \mu_{0}$. The PEC region is denoted $R_{2}$. In a plane perpendicular to the edge of the coated wedge, the boundary between the regions $R_{0}$ and $R_{1}$ will be denoted $S_{10}$. Similary, the boundary between the regions $R_{1}$ and $R_{2}$ will be denoted $S_{21}$. The unit normal vector $\hat{n}_{10}$ to $S_{10}$ is oriented into the region $R_{0}$. We introduce a rectangular $(x, y, z)$ coordinate system and a circular cylindrical $(\rho, \varphi, z)$ coordinate system with the $z$-axis coinciding with the 
edge of the PEC wedge. In this coordinate system, the observation point $P$ is specified by the coordinates $(\rho, \varphi)$. A $z$-independent uniform plane wave $\left(E^{i}, H^{i}\right)$ is impinging upon the coated wedge and we specify the polarization of this plane wave to be TM with respect to z. The direction of propagation of $\left(\mathrm{E}^{i}, \mathrm{H}^{\mathrm{i}}\right)$ forms an angle $\varphi^{i}$ with the 8 -axis. The field in the region $R_{0}$ will be denoted $\left(E_{0}, H_{0}\right)$ and the field in the region $R_{1}$ will be denoted $\left(E_{1}, H_{1}\right)$.

We apply the surface equivalence principle [8] to derive three coupled integral equations from which three unknown quantities can be found. These are $J_{10}$ and $\mathbf{E}_{10}$ (an equivalent electric surface current density on $S_{10}$ and the electric field on $S_{10}$ in the case where the field in the region $R_{0}$ equals $\left(E_{0}, H_{0}\right)$ and the field in the regions $R_{1}$ and $R_{2}$ equals $(0,0)$ ) and $J_{21}$ (the induced electric surface current density on $S_{21}$ ). All these quantities are 2 -directed. The three coupled integral equations are (time variation $e^{j \omega t}$ is assumed and suppressed)

$$
\begin{aligned}
-E^{i}(\bar{\rho})= & -\frac{k_{0} \zeta_{0}}{4} \int_{s_{10}} J_{10}\left(\bar{\rho}^{\prime}\right) H_{0}^{(2)}\left(k_{0}\left|\bar{\rho}-\bar{\rho}^{\prime}\right|\right) d s^{\prime}-\frac{E_{10}(\bar{\rho})}{2} \\
& +\frac{j}{4} \int_{s_{10}} \nabla \times\left(H_{0}^{(2)}\left(k_{0}\left|\bar{\rho}-\bar{\rho}^{\prime}\right|\right) E_{10}\left(\bar{\rho}^{\prime}\right) \times \hat{n}_{10}\right) d s^{\prime}, \quad \bar{\rho} \in S_{10} \\
0= & \frac{k_{1} \zeta_{1}}{4} \int_{s_{10}} J_{10}\left(\bar{\rho}^{\prime}\right) H_{0}^{(2)}\left(k_{1}\left|\bar{\rho}-\bar{\rho}^{\prime}\right|\right) d s^{\prime}-\frac{E_{10}(\bar{\rho})}{2} \\
& -\frac{j}{4} \int_{s_{10}} \nabla \times\left(H_{0}^{(2)}\left(k_{1}\left|\bar{\rho}-\bar{\rho}^{\prime}\right|\right) E_{10}\left(\bar{\rho}^{\prime}\right) \times \hat{n}_{10}\right) d s^{\prime} \\
& -\frac{k_{1} \zeta_{1}}{4} \int_{S_{21}} J_{21}\left(\bar{\rho}^{\prime}\right) H_{0}^{(2)}\left(k_{1}\left|\bar{\rho}-\bar{\rho}^{\prime}\right|\right) d s^{\prime}, \quad \bar{\rho} \in S_{10} \\
0= & \frac{k_{1} \zeta_{1}}{4} \int_{s_{10}} J_{10}\left(\bar{\rho}^{\prime}\right) H_{0}^{(2)}\left(k_{1}\left|\bar{\rho}-\bar{\rho}^{\prime}\right|\right) d s^{\prime}, \\
& -\frac{j}{4} \int_{s_{10}} \nabla \times\left(H_{0}^{(2)}\left(k_{1}\left|\bar{\rho}-\bar{\rho}^{\prime}\right|\right) E_{10}\left(\bar{\rho}^{\prime}\right) \times \hat{n}_{10}\right) d s^{\prime} \\
& -\frac{k_{1} \zeta_{1}}{4} \int_{S_{21}} J_{21}\left(\bar{\rho}^{\prime}\right) H_{0}^{(2)}\left(k_{1}\left|\bar{\rho}-\bar{\rho}^{\prime}\right|\right) d s^{\prime}, \quad \bar{\rho} \in S_{21}
\end{aligned}
$$

where $\mathrm{k}_{\mathrm{i}}$ and $\zeta_{\mathrm{i}}(\mathrm{i}=0,1)$ denote the wave number and the intrinsic impedance, respectively, of the region $\mathrm{R}_{\mathrm{i}}, \mathrm{H}_{0}{ }^{(2)}$ denotes the Hankel function of zero order and second kind and the '.' on an integral sign denotes a principal value integral. When the three unknown quantities are known, the scattering by the coated wedge illustrated in Fig. 1 is easily determined via radiation integrals involving $\mathbf{J}_{10}$ and $\mathbf{E}_{10}$. 


\section{NUMERICAL SOLUTION OF INTEGRAL EQUATIONS}

The integral equations (1)-(3) for the coated wedge are solved by a hybrid technique in line with the approach of Burnside et al [9] for the PEC wedge. Close to the edge of the wedge, the unknown quantities are expanded via the use of pulse expansion functions of width $\lambda_{0} / 20$, where $\lambda_{0}$ denotes the free space wavelength. Away from the edge of the wedge, the unknown quantities are assumed to be built up of a PO component, a fringe wave (FW) component and a SW component. Since the PO components as well as the decay factors and the propagation constants for the FW and SW components are known, each unknown quantity can be expressed in terms of two unknown complex constants on each wedge face. This expansion of the unknown quantities allow for the pulse expansion region to be as small as $1.5 \lambda_{0}$ on each face of the PEC wedge. Exclusion of the FW term would require a much larger pulse expansion region and hereby a significantly larger total number of unknowns. Dirac delta functions are used as weighting functions and the resulting equation system is solved by use of the conjugate gradient method [10]. All integrals over infinite intervals are evaluated by use of the method of averages [11].

\section{NUMERICAL RESULTS}

Since we will investigate whether or not an edge in a coated structure makes the SIBC approximation inapplicable, it must be required that the SIBC approximation be accurate when the edge is not present, i.e. for an infinite coated plane. We choose the relative permittivity $\epsilon_{\mathrm{r}}=6-\mathrm{j} 6$, the relative permeability $\mu_{\mathrm{r}}=1$ and coating thicknesses less than $\mathrm{d}=0.15 \lambda_{0}$.

We consider wedge coatings with the thicknesses $d=0.05 \lambda_{0}$ and $d=0.15 \lambda_{0}$. For neither coating does unattenuated SW propagation occur. The angle of incidence $\varphi^{\mathrm{i}}$ equals $-\pi / 2$. Echo width (normalized to the free space wavelength) as a function of the observation angle $\varphi$ for both coated, SIBC and PEC wedges are shown in Fig. 2(a-b). Geometrical optics contributions (direct and reflected fields) have been extracted so only the edge diffraction remains. The results for both SIBC and coated wedges predict the echo width to be zero for $\varphi= \pm 135^{\circ}$ (on the wedge faces) as well as peaks at the reflection boundary $\left(\varphi=0^{\circ}\right)$ and the shadow boundary $\left(\varphi=90^{\circ}\right)$. For the coating thickness $d=0.05 \lambda_{0}$, the SIBC wedge is seen to provide an accurate approximation. For the coating thickness $d=0.15 \lambda_{0}$, a discrepancy can be observed for observation angles less than $-50^{\circ}$. For observation angles exceeding $-100^{\circ}$, this discrepancy is less than $2 \mathrm{~dB}$ while it increases to $4 \mathrm{~dB}$ for observation angles less then $-100^{\circ}$. The result for the uncoated PEC wedge is shown to illustrate the effect of the wedge coatings.

\section{CONCLUSION}

The SIBC approximation is not formally applicable to coated structures with edges. However, the results presented here revealed that for certain coatings, the SIBC approximation yields accurate results. Hence, the presence of an edge in a coated structure does not necessarily make the SIBC approximation inapplicable. However, the SIBC approximation is inaccurate in some cases [12]. For instance, for a lossless coating where unattenuated SW propagation can take place $\left(\epsilon_{\mathrm{r}}=5, \mu_{\mathrm{r}}=1, \mathrm{~d}=0.15 \lambda_{0}\right)$, the diffraction by the SIBC wedge was found to give an inaccurate description of the diffraction by the coated wedge. Due to the many parameters for the coated wedge configuration, the range of validity for the SIBC approximation can only be obtained through an extensive numerical investigation. The analysis method described here provides an efficient tool to this end. It is noted that the method is readily applicable to incident fields other than plane waves. It can easily be accomodated to TE illumination and the extension to multilayer coatings is straightforward. 